\title{
Bibliographie critique des Modèles de Prévision des Effectifs étudiants au niveau universitaire
}

\author{
GILLES GUERIN*
}

\section{Introduction}

L'objectif de cet article est de passer en revue les différents modèles de prévision des effectifs étudiants au niveau universitaire. De telles entreprises ont déjà été réalisées. Citons notamment et par ordre chronologique: Wurtele Z. (63), Weitz H. $(58,59)$, Baisuck A. (3), Weathersby G. et Weinstein M. (57), Florian M. et Guérin G. (21). "Même dans les pays où elle a un semblant d'existence, cette forme de planification constitue un développement très récent ... qui a revêtu l'aspect d'une véritable révolution" (53). Comme dans toutes les révolutions, on assiste à une progression très rapide dans ce domaine. Des bibliographies sur ce sujet, comme celles de Wurtele et Weitz sont déjà partiellement démodées seulement après six années dans un cas et quatre dans l'autre. Des groupes de recherche tels que "I'Institute for the Quantitative Analysis of Social and Economic Policy" de Toronto ou l' "Office of the Vice President Planning and Analysis" de Berkeley ont publié dans cet intervalle de temps à une telle cadence qu'un grand nombre de leurs recherches répondent déjà plus ou moins aux problèmes soulevés dans les recherches présentées par les auteurs ci-dessus. La classification et la critique des modèles de prévision des effectifs sont originales et constituent, même si elles peuvent s'avérer mal fondées ultérieurement, une pensée personnelle basée sur des réflexions et des lectures antérieures à la date de rédaction de cet article.

\section{Modèles de prévision des effectifs étudiants}

\subsection{Modèles purement projectifs}

Sans se préoccuper de la structure du système universitaire ni des flux d'étudiants à l'intérieur de celui-ci il est possible de relier l'indice de fréquentation d'une population étudiante à des caractéristiques telles que l'âge, le sexe, le cycle d'étude, le type d'enseignement, la localisation géographique ou les caractéristiques socio-économiques. Une telle relation établie, il est alors possible de déduire les effectifs étudiants des projections des indices de fréquentation et des populations futures ayant la même caractéristique.

\footnotetext{
* Gilles Guérin est Professeur adjoint, Ecole des Relations Industriclles, Université de Montréal.
} 
Lorsque le choix des caractéristiques définissant la population est tel que les tendances sont bien définies, cette méthode est précieuse et permet d'estimer les effectifs un grand nombre de périodes à l'avance. C'est le cas pour les populations définies en fonction de l'âge ou du sexe $(15,30)$; à l'aide de projections démographiques, on peut élaborer des projections complètes des effectifs étudiants. Généralement ces projections sont réalisées à un niveau agrégé compatible avec les données démographiques. Pourtant il est possible d'effectuer ces projections au niveau d'une région, d'une institution ou même d'un programme d'enseignement $(28,35,44,50,51)$. Dans ce dernier cas Smith W. $(50,51)$ et ensuite O'Grady W. (44) classent chaque étudiant en fonction du statut (nouveau, actif ou inactif) et dù niveau d'avancement dans le programme. Ayant projeté les populations futures d'étudiants ils supposent ensuite que la structure des admissions et des transitions d'un groupe à un autre est identique à celle de la période précédente et estiment ainsi les effectifs des différents groupes. L'hypothèse de travail de Smith $W$. ne peut s'appliquer malheureusement qu'à une institution universitaire telle que l'Université de Californie à Los Angeles qui a atteint sa capacité maximale et de ce fait même présente un certain lissage des flux d'étudiants comparable aux variables démographiques. D'ailleurs même dans ce cas les projections n'excèdent pas un ou deux semestres. Dans d'autres modèles $(17,22,31,43,62)$ la population est représentée par les gradués du secondaire désireux de poursuivre des études supérieures. Les caractéristiques de cette population sont l'âge, le sexe etc.... mais surtout la capacité à payer les frais de scolarité et indirectement le montant de l'aide nécessaire à l'étudiant pour équilibrer son budget. En projetant la population susceptible d'entrer dans le système universitaire et les proportions d'étudiants dans chaque classe d'aide, Jewett J. (31) détermine les admissions dans le système universitaire compatibles avec un certain budget.

Les principaux avantages que l'on peut dégager de ces modèles de projection sont les suivants.

Les données sont peu nombreuses et relativement simples à obtenir. Les données démographiques, généralement disponibles, peuvent être utilisées.

Les techniques utilisées: régressions, lissages, etc. . . sont facilement compréhensibles et ne nécessitent pas des moyens de calcul ni des délais importants.

L'étude-comparative des indices de fréquentation peut permettre de porter un avis sur les conditions d'enseignement ou sur le degré de réalisation des objectifs.

Par ailleurs l'usage de ces modèles présente un certain nombre d'inconvénients.

Le principal est relatif à la méconnaissance des flux et de la structure interne du système. Cet inconvénient est d'autant plus préjudiciable que le niveau d'agrégation est élevé.

A un niveau désagrégé, les projections des populations concernées, surtout si elles ne sont pas décrites par des caractéristiques démographiques, peuvent présenter une faible fiabilité qui se répercute sur l'estimation des effectifs.

Ces modèles sont statiques; ils n'expliquent pas la cause des valeurs projetées et ne donent aucune précision sur leurs modifications possibles. Aucun contrôle du système n'est possible par l'intermédiaire de ces modèles purement représentatifs. 
Si l'on utilise les valeurs projetées dans un but comparatif, des indices de fréquentation différents ne reflètent pas forcément des différences entre les efforts ou conditions d'instruction puisque les indices sont liés à la structure des caractéristiques choisies pour décrire la population.

\subsection{Modèles en chaine de Markov}

Si l'évolution dans un système peut être décrite en termes probabilistes, le processus sera aléatoire. Supposons que la connaissance de l'état du système aux instants consécutifs $1,2 \ldots t$ antérieurs à $t+1$ apporte bien quant à la connaissance de son état en $t+1$ une certaine information mais tout entière contenue dans la connaissance de son état à l'instant le plus récent $\mathrm{t}$. "Autrement dit tout le passé de l'évolution du système se trouve résumé dans son état au dernier instant où on le connaît. Une évolution possédant cette propriété s'appelle processus de Markov" (24). Un tel processus de Markov s'il est aléatoire et si l'espace des états est un ensemble fini ou dénombrable s'appelle une chaîne de Markov discrète $\left(x_{t}\right)$. La probabilité que $x_{t+1}$ soit dans l'état $j$ étant donné que $x_{t}$ est dans l'état i (appelée probabilité de transition à une étape) est notée $p_{i j}{ }^{t, t+1}$ c'est-à-dire que l'on a $\mathrm{P}_{\mathrm{ij}}{ }^{\mathrm{t}, \mathrm{t}+1}=\operatorname{Pr}\left(\mathrm{x}_{\mathrm{t}+1}=\mathrm{j} / \mathrm{x}_{\mathrm{t}}=\mathrm{i}\right)$. Lorsque les probabilités de transition à une étape sont indépendantes de la variable temps on dit que le processus de Markov est à probabilités de transition stationnaires (32).

On voit immédiatement l'intérêt des chaînes de Markov si on considère le système universitaire comme une structure où des transitions peuvent se produire, à des instants bien déterminés, entre les différents états de cette structure. Si l'on connaît ses probabilités de transition entre les états, l'évolution d'un étudiant dans le système sera une chaîne de Markov discrète. De tels modèles sont alors des micro-modèles centrés sur le comportement individuel. La connaissance de la matrice des probabilités de transition et de son état à l'instant $\mathrm{t}$ résume l'histoire passée de l'individu et toutes ses caractéristiques personnelles. Si l'on veut appliquer ce modèle à un groupe d'individus, il faut supposer que l'homogénéité du groupe est telle que la matrice des probabilités de transition s'applique à chacun d'aux. Si tel n'est pas le cas, l'hypothèse markovienne ne peut être conservée. Si elle l'est malgré tout, c'est souvent pour bénéficier de l'élégance de la formulation des chaines de Markov et de toute la théorie qui a été élaborée et qu'il suffit d'appliquer au système universitaire $(24,32)$. Dans ce cas il serait bénéfique d'estimer l'erreur introduite par une telle approximation. Y a-t-il convergence ou non de l'erreur vers zéro lorsque le niveau d'agrégation des données est important $(9,20)$ ? Baisuck A. (3) fournit une illustration de cette erreur dans le but de rejeter l'hypothèse markovienne. Il vérifie qu'une matrice prise comme moyenne pondérée de deux matrices de probabilités de transition n'a pas une matrice-limite qui est la moyenne pondérée des deux matriceslimites de probabilité de transition. Bien que les deux matrices de probabilité soient assez dissemblables et le niveau des données assez désagrégé, les différences introduites dans l'évolution de deux structures relatives aux deux méthodes, même au bout d'un grand nombre de périodes, ne sont pas convaincantes. Néanmoins les auteurs qui utilisent cette approche doivent toujours exprimer une certaine faiblesse au niveau des hypothèses sans pouvoir quantifier la conséquence sur leurs résultats ou conclusions d'une telle approximation. Ainsi Thonstad T. (55): "L'application différera de la méthode briève- 
ment décrite ci-dessous en ce que nous utiliserons un modèle déterministe au lieu de la méthode stochastique. Cela signifie que nous interpréterons les probabilités de transition comme des coefficients de transition fixes, et non comme des probabilités". Cette interprétation est ambigue puisque le terme chaîne de Markov que Thonstad T. utilise dans la suite de sa recherche, se réfère, par définition, à un processus aléatoire. De même citons Marshall K. et Oliver R. (36). "It has the appealing feature that the fractions $P_{j}$ can be interpreted as transition probabilities and thus allows one to adopt useful results from the theory of Markov chains even though the process itself may not be Markovian" et Marshall K., Oliver R. and Suslow S. (37): " $\mathrm{P}_{\mathrm{ij}}$ can be interpreted as a probability defined for an individual student or a fraction defined for a large number of students".

Les premières suggestions concernant ce type de modèle ont été faites par Gani J. (23) quoique son modèle soit un modèle de projection déterministe, et par Young $\mathrm{A}$. et Almond G. (64) puis par Bartholomew D. (5). Dans ces deux derniers cas, les modèles sont relatifs à un système de main d'oeuvre plutôt qu'au système universitaire. Bartholomew D. dans son livre analyse toutes les implications sur le modèle de la théorie des chaines de Markov. Par la suite Oliver R. (45), puis Marshall K. et Oliver R. (36) et Marshall K., Oliver R. et Suslow S. (37) ont sophistiqué le modèle et l'ont surtout testé avec des données réelles de l'université de Californie à Berkeley pour la période 1955 1956. Tous ces auteurs supposent que la matrice des probabilités de transition est stationnaire dans le temps, tout au moins pour des horizons de moyenne période, que Gani J. (23) fixe à environ cinq ans. Cette hypothèse, qui n'est pas évidente, est toujours acceptée puisque son rejet entrainerait le choix d'un autre type de modèle étant donné que la théorie des chaînes de Markov à probabilités de transition non stationnaires présente un intérêt pratique restreint. La plupart de ces modèles assimile le système à une structure progressive fonction des cycles et des années d'étude. Cette structure reflète de plus en plus mal la structure des universités nord-américaines qui évoluent vers des structures plus souples et plus variées. Dans cette optique Marshall K. et Oliver R. (36) caractérisent les états de la structure en fonction du statut de l'étudiant: actif, inactif, éliminé, gradué, mais là encore ils distinguent les actifs en fonction du cycle d'étude et de l'année d'étude. Or dans le contexte de plus en plus général d'une progression par matière il devient vite impossible et superflu d'identifier l'étudiant à une année d'étude. Il importe seulement de connaitre le nombre et l'articulation des cours nécessaires à l'obtention du grade que l'étudiant cherche à obtenir suivant son rythme personnel et ses réussites antérieures. Dans cette optique Turksen I. (56) propose une structure relative à l'activité plutôt qu'au programme ou au système. Bien que le modèle de Turksen I. soit un modèle de simulation, on peut penser que ce micro-modèle pourrait se présenter suivant une formulation markovienne où chaque étudiant dans le système se trouverait vis-à-vis de chaque cours proposé dans un des trois états suivants proposés par Turksen I.: éligible, non-éligible car la structure des prérequis n'est pas satisfaite, non-éligible car le cours a déjà été suivi avec succès. Pour chaque combinaison cours-étudiants les hypothèses des chaines de Markov sont rigoureusement vérifiées puisque les probabilités de transition reflètent les caractéristiques de l'étudiant, du cours et des interactions étudiant-professeur. En ce sens Turksen I. propose un des rares modèles qui esquissent une étude du système en tenant compte de certains éléments du processus éducatif. C'est un pas vers la synthèse des 
micro-modèles et des macro-modèles que réclame Correa $H$. (15). Malheureusement, du point de vue opérationnel, ce modèle requiert l'estimation d'un nombre incroyable de paramètres. Dans le but d'une agrégation raisonnable des données on retrouve alors les hypothèses simplificatrices, habituelles aux modèles de cette classe, classées suivant le niveau d'agrégation désiré: les comportements des étudiants d'un programme d'étude vis-à-vis d'un cours particulier sont identiques; les comportements des étudiants d'un même programme d'étude sont identiques; les comportements des étudiants en général sont identiques. L'adoption de l'une ou l'autre de ces deux dernières hypothèses nous ramène aux modèles précédemment décrits. La première hypothèse est plus originale et dans le contexte d'une progression par matière il semble qu'elle puisse engendrer des prévisions fructueuses au niveau désagrégé le plus raisonnable.

Les principaux éléments positifs des modèles en chaîne de Markov peuvent se résumer ainsi.

Toute la théorie des chaînes de Markov discrètes d'ordre 1 peut être utilisée. En voici énumérées quelques unes des propriétés les plus importantes relativement à leur application au système universitaire: les probabilités de passage d'un état à un autre de la chaîne en $t$ périodes sont données par la matrice puissance $t$-ième de la matrice des probabilités de transition; d'après le théorème de Sylvester il est possible de décomposer cette matrice à la puissance $t$-ième en une somme de produits des valeurs propres de la matrice de probabilité par des matrices orthogonales entre elles. Cette somme représente la décomposition spectrale de la matrice à la puissance t-ième; la donnée de la loi de probabilité initiale (qui est généralement la connaissance de la structure) et de la matrice des probabilités de transition suffit à déterminer la loi de probabilité à tout instant ultérieur t. Dans un système fermé on obtient l'espérance des effectifs dans chaque état de la structure au temps t en multipliant le nombre d'individus dans le système par cette loi de probabilité au temps t. Dans un système ouvert il faut additionner les admissions espérées dans chaque état de la structure pour cette même période; la loi de probabilité au temps t peut tendre pour $t \rightarrow \infty$ vers une distribution de probabilité stationnaire, indépendante de la loi de probabilité initiale. Il est nécessaire et suffisant pour que la distribution de probabilité stationnaire soit indépendante de la loi de probabilité initiale que la matrice puissance $\mathrm{t}$-ième de la matrice des probabilités de transition tende vers une matrice-limite, stochastique et ayant toutes ses lignes identiques entre elles et de plus identiques à la distribution de probabilité stationnaire.

Ces modèles étant probabilistes, il est possible d'estimer les variances et covariances des effectifs dans les états de la structure. De telles estimations, quoique difficilement maniables et basées sur des hypothèses très restrictives, ont été réalisées par Bartholomew D. (5).

On peut introduire dans le modèle un certain degré de non-homogénéité représenté par des probabilités d'admission et de transition dans le système variables suivant les différentes sessions de l'année. Les propriétés des chaînes de Markov sont conservées si l'on suppose que les probabilités sont homogènes dans le temps relativement a chacune des sessions. 
Gani J. (5, pp. 55-58) dans son étude relative à l'Université d'Etat du Michigan distingue ainsi trois matrices de probabilité de transition et trois vecteurs de probabilité d'admission relatifs aux trois trimestres de l'année académique.

La classification des états d'une chaîne de Markov en transitoires ou absorbants s'applique particulièrement bien aux états de la structure du système universitaire qui sont tous transitoires et aux états de sortie définitive du système qui sont absorbants. Elle permet notamment d'étudier ce phénomène d'abandon momentané des études qui devient de plus en plus important dans les universités. Cet état d'inactivité est un état temporaire pour ceux qui vont revenir dans le système un certain nombre de périodes plus tard.

Il est possible de distinguer les probabilités de premier passage des probabilités de passage simple conformément à la théorie des chaines de Markov. L'intérêt d'une telle distinction dans le cas d'une structure par grade et par année d'étude est faible mais dans le cas d'une structure fonction du statut de l'étudiant (actif, inactif, gradué) cette distinction est plus fructueuse.

Enfin du point de vue conceptuel, l'introduction de chaines de Markov d'ordre limité mais supérieur à 1 serait plus satisfaisante. La théorie des chaînes de Markov pourrait alors s'appliquer à des groupes d'individus ayant un certain nombre de caractéristiques communes alors qu'actuellement on présume de l'homogénéité à partir uniquement d'une caractéristique qu'elle soit l'année d'étude ou le statut de l'étudiant. Malheureusement la manipulation de ces chaînes d'ordre supérieur à 1 est extrêmement encombrante et malcommode.

On peut ramener les inconvénients de ces modèles aux points suivants:

Une chaîne de Markov étant un modèle stochastique, l'hypothèse markovienne ne peut être vraie que pour un seul individu ou un petit groupe très homogène. Les modèles qui utilisent cette approche devraient donc être des micro-modèles. Or certains auteurs (36, $54,55)$ utilisent la théorie des chaines de Markov pour des macro-modèles sans pouvoir estimer le niveau d'erreur introduit par une telle approximation.

Les modèles en chaîne de Markov décrivent le cheminement des individus à l'intérieur du système. Ils ne donnent aucune indication sur l'évaluation des ressources nécessaires à cette progression dans la structure.

Comme le remarque Thonstad T. (54) le modèle en chaîne de Markov permet de rester, dans un état transitoire pendant plus de deux périodes. Même si la probabilité d'un tel état de chose va en décroissant, cela est irréaliste dans le cas d'une structure par année d'étude par exemple.

Les probabilités de transition sont supposées homogènes ce qui dans certains cas est une assez grossière approximation (au niveau gradué notamment ou pour des prévisions supérieures à une période). Une étude comparable à celle de Breneman D. (9) sur la stabilité des coefficients utilisés dans les modèles de répartition des charges de travail du corps professoral serait très utile et nous permettrait de tester la validité de l'hypothèse d'homogénéité. D'ailleurs il semble que la stabilité des probabilités de transition dépende des conditions particulières à chaque institution. Admettre l'hypothèse de stabilité de Gani, même 
pour des horizons inférieurs à cinq ans, pourrait être discutable dans un grand nombre de cas particuliers.

Même dans le cas d'une quasi-stabilité des probabilités de transition, la sensibilité des modèles par rapport à de faibles variations des probabilités de transition est généralement ignorée? Des variations insignifiantes de ces probabilités ne peuvent-elles avoir des conséquences inattendues sur le comportement-limite des distributions de probabilité dans les états de la structure?

Enfin la plupart des modèles en chaine de Markov ne comprennent aucun traitement explicite de l'estimation des probabilités de la matrice de transition. Beaucoup de facteurs autres que les données historiques: bourses, frais de scolarité, salaire espéré, chômage, restrictions à l'admission etc. . . . peuvent influencer ces probabilités et du point de vue appliqué leur détermination est cruciale, puisqu'elles représentent le seul aspect auquel s'attache l'analyse du système.

\subsection{Modèles markoviens déterministes}

L'approche est semblable aux modèles en chaîne de Markov si ce n'est que les auteurs "deliberately avoid talking about transition probabilities, because that approach rests on certain implausible assumptions concerning the identical behaviour of individuals of the population, and because it leads to certain intractable problems in probability theory and in statistical estimation" (13). A part l'option déterministe, la description de la structure et la définition des états sont identiques aux modèles proposés en 2.2. Puisqu'elle n'est plus une condition nécessaire à l'application du modèle, la condition d'homogénéité dans le temps des proportions de transition est généralement rejetée. Les modèles de cette classe sont libérés de la rigueur de la formulation en chaîne de Markov et il est possible de les ajuster aux caractéristiques particulières du système en ajoutant aux contraintes relatives à la progression dans la structure des contraintes secondaires relatives aux capacités des différents états, aux limites d'admission, au développement désiré, aux proportions d'étudiants dans les états etc. ... Une formulation initiale de ce type de modèle est due à Moser C. et Redfern P. (40). Ce modèle a ensuite été repris par Clough D. (12), Armitage P. et Smith C. (1), Price W. (47) et Guérin G. (25, 26, 27). Le modèle de Clough D. (12) contient des contraintes sur l'offre de places dans le système (fonction des standards, locaux, ratios, professeurs, budgets etc.) et des contraintes sur la demande de places (14, 41) dans ce même système (fonction des frais de scolarité, coût de la vie, durée des études, salaires espérés, etc.). L'ensemble de ces contraintes détermine un ensemble convexe dont la fermeture varie au gré des décisions administratives et à l'intérieur duquel se situent les proportions de transition réalisables. Une fonction économique relative au bénéfice social, que Clough choisit de maximiser, permet de sélectionner l'ensemble optimal des proportions de transition pour un horizon de une période. Pour un horizon plus éloigné, le modèle n'est plus linéaire et il est suggéré d'employer la programmation dynamique pour optimiser la fonction économique. Ce modèle est très élégant mais il présente l'inconvénient de nécessiter une somme de données importante pour la construction des contraintes. Notamment pour les contraintes de demande il est déjà bien difficile de déceler l'influence d'un seul facteur $(29,60,62)$ ! La simulation de Baisuck A. $(2,3,4)$ utilise le même type de modèle. Des regressions permettent la projection des effectifs dans la 
structure. Dans le cas d'objectifs à atteindre, les projections sont modifiées, d'une façon progressive ou bien unique afin de réaliser ces objectifs. Le modèle de Dietze H. (16) quoique adapté à la rigidité des systèmes universitaires européens, utilise la même matrice de proportions de transition ce qui lui permet de prévoir les effectifs dans le système au cours de la période suivante à partir de ceux dans le système actuellement. A partir des taux de participation des étudiants aux différents cours et du nombre maximum d'étudiants à ces mêmes cours, l'auteur détermine les admissions dans les différents programmes d'étude et par la même occasion identifie les goulots d'étranglement dans la structure. Caspar R. (10, 11) propose un modèle identique mais utilise la méthode du simplexe pour déterminer la structure optimale des admissions. Dans le modèle de Dietze $H$. on peut se demander qui va déterminer le nombre maximum d'étudiants pour chaque cours car la détermination de ces capacités n'est liée à aucune autre variable et reste à la discrétion du planificateur. De plus ces deux modèles correspondent à une conception assez figée de l'enseignement supérieur; ils déterminent l'arrangement optimal des étudiants que l'on peut accepter dans le système avec les ressources qui existent. Ainsi le corps professoral détermine l'offre de places dans le système et la demande étudiante doit se modifier pour s'adapter à la structure des enseignants et réduire les goulots d'étranglement. Dans le contexte américain, cette approche est irréaliste car la demande des étudiants a une très grosse influence sur l'offre de places dans le système; de plus la structure des enseignants peut évoluer et se modeler beaucoup plus qu'en Europe puisque les admissions y sont simples et les départs fréquents.

Comme dans les cas précédents, on peut énumérer les avantages suivants.

Suivant cette approche les auteurs utilisent les techniques qui leur semblent appropriées pour résoudre un problème donné. Suivant l'approche en chaîne de Markov, on a parfois l'impression d'une recherche d'exemples appropriés à l'application d'une technique donnée soit les chaînes de Markov.

Contrairement aux modèles en chaine de Markov, les modèles de ce groupe peuvent utiliser des techniques d'optimisation. Alors que les premiers prévoient la progression des étudiants dans la structure, les seconds peuvent indiquer les modifications optimales de cette progression ou bien déterminer les admissions adéquates en vue d'atteindre certains objectifs.

Face à la rigidité conceptuelle des chaînes de Markov, ces modèles s'avèrent beaucoup plus souples et peuvent répondre plus facilement aux exigences particulières de certaines institutions universitaires.

Il n'y a pas de restriction quant au niveau d'agrégation des variables du système.

Les inconvénients suivants peuvent être énoncés.

Dans l'optique habituelle de la non-homogénéité des matrices de proportions de transition, les modèles utilisant des techniques d'optimisation sont généralement peu explicites pour des horizons supérieurs à une période. Dans beaucoup de cas les contraintes du programme d'optimisation ne sont plus linéaires.

La description du système universitaire étant relativement partielle dans ces modèles, on 
aboutit généralement pour les modèles d'optimisation à des sous-optimisations relativement à l'ensemble des variables du système.

Le comportement des étudiants dans la structure est un comportement stochastique. Le décrire en termes déterministes est une approximation.

\subsection{Modèles d'entrée-sortie}

Toujours en fonction d'une progression des modèles du plus simple au plus sophistiqué, du plus descriptif au plus explicatif, cette dernière classe de modèles applique au système universitaire le modèle de production généralement appelé modèle d'entrée-sortie (ou in put-output). Ce modèle est déjà plus qu'un simple modèle de prévision d'effectifs étudiants, il représente possiblement un modèle quasi-global du système et pourrait se ranger aussi bien parmi les modèles de prévision de ressources, d'allocation de ces ressources ou surtout parmi les modèles d'évaluation des programmes d'enseignement.

Dans le cas le plus général, il existe une transformation linéaire qui lie le vecteur d'entrée au vecteur de sortie. Si les sorties correspondent aux "produits finis" du système de production universitaire, les entrées seront alors toutes les ressources nécessaires à la production de ces sorties soit des nouveaux étudiants, des professeurs, du personnel de soutien, des locaux, du matériel etc. ... Stone R. (52) distingue trois grandes catégories dans ces entrées du système: les étudiants, la main d'oeuvre, les locaux et l'équipement. Les coefficients de la matrice d'entrée-sortie indiquent les décisions prises par les administrateurs et les restrictions du système. Ils reflètent les contraintes sur les rapports étudiants-professeur, sur les répartitions de charges de cours, sur l'affectation des professeurs, etc. ... Si l'on précise un vecteur de sortie désiré, ils permettent de déterminer les entrées nécessaires à la réalisation des objectifs. Les modèles de cette classe ne se présentent pas tous sous la forme matricielle que l'on vient de décrire; certains $(8,38)$ correspondent à des systèmes d'équations linéaires représentant les contraintes imposées à certaines ressources pour la production des sorties. Il suffirait de réarranger les équations et d'introduire quelquefois certaines ressources supplémentaires pour avoir un modèle complet d'entrée-sortie. En effet la plupart des modèles $(8,38,42,46,48)$ se limitent aux ressources humaines et ne se préoccupent que des sorties représentées par les étudiants gradués. Par le biais de capacités, relatives à chacun des cours offerts, le modèle de Braun H., Hammer G. et Schmid K. (8) tient compte des salles de classe et du matériel mais la relation n'est pas explicite. Comme le modèle de Menges G. et Elstermann G. (39) ainsi que ceux précédemment décrits de Dietze $(16)$ et Caspar $(10,11)$, ce modèle européen détermine les admissions possibles en fonction d'un arrangement donné et d'une utilisation maximale de certaines ressources, professeurs et/ou locaux et non en fonction d'objectifs sur les sorties ou les entrées d'étudiants comme le font généralement les auteurs nord-américains. Parmi ceuxci, Nordell L. (42) et Oliver R., Hopkins D. et Armacost R. (46) se préoccupent uniquement des effectifs d'étudiants et d'enseignants. Contrairement aux modèles allemands il s'agit ici de déterminer le nombre d'enseignants nécessaires à la production d'une structure désirée de gradués. De la même manière le modèle de Lawson D. et Jewett F. (34) appliqué au Collège d'Etat de Humboldt permet en fonction de la structure de gradués de générer l'ensemble des ressources physiques et humaines nécessaires. A un niveau intermédiaire de généralisation se situent des modèles tels que ceux de Koenig H. (33) qui 
transforment toutes les ressources humaines à l'entrée du système, y compris le personnel de soutien, en sorties réparties suivant les fonctions primaires d'enseignement, de recherche, de service au public et même la fonction administrative d'appui. Enfin, au niveau le plus général, le modèle comprend toutes les ressources à l'entrée du système, qu'elles soient humaines ou matérielles, et produit des gradués, des connaissances et des services. De tels modèles $(6,49,52)$ s'inscrivent généralement dans un contexte plus vaste encore, où le système éducatif est considéré comme un des secteurs de l'économie. Ces modèles sont alors tournés vers la détermination des ressources nécessaires à la production d'une certaine structure de gradués nécessaire au développement économique et social. Redfern P. (49) conçoit le système éducatif comme une industrie qui fournirait comme matières premières aux autres industries les personnes qualifiées nécessaires: "We may treat the educational system as an industry or group of industries producing manpower with a range of skills; treat young people as the "intermediate products" moving from one part of the educational system to another; and treat the rest of the economy outside the educational system as the final recipient of the products of the educational system". A partir des mêmes hypothèses Bénard J. (6) introduit le concept d'optimisation relativement à l'effectation "des ressources, pour la plupart physiques, entre l'activité d'éducation et les activités économiques marchandes, représentées par les secteurs d'un tableau d'échange inter-industriel. L'optimisation est obtenue par la maximisation sous contraintes d'une fonction sociale de préférence, elle-même constituée par des indicateurs numériques du niveau de vie de la population tout au long de la période pluriannuelle envisagée, et du potentiel de production en fin de période".

Voici, en résumé, les avantages principaux de ces modèles.

Le modèle d'entrée-sortie est un modèle global qui évalue les ressources nécessaires à la production de sorties. Contrairement aux modèles précédents qui étaient en grande partie descriptifs, ce modèle étudie les relations internes et reflète une analyse complète du système.

Une fois élaboré, ce modèle représente un outil de gestion utile pour l'administrateur. La sensibilité des sorties à certaines ressources particulières, la modification des coefficients de la matrice, la substitution de certaines ressources peuvent être testées.

Le modèle d'entrée-sortie est linéaire et sa souplesse conceptuelle permet l'adjonction de contraintes supplémentaires; de la même façon il peut être intégré au milieu d'un système matriciel plus large. Des techniques d'optimisation linéaire peuvent être utilisées très facilement.

Les résultats de la théorie économétrique des modèles d'entrée-sortie de Léontief W. (18) peuvent être appliqués aux modèles d'entrée-sortie du système universitaire. Cette théorie permet l'utilisation comme ressources de certaines sorties du système; dans le système universitaire ceci peut s'appliquer aux étudiants gradués qui ont une fonction d'encadrement des étudiants sous-gradués ainsi qu'aux gradués au niveau du doctorat qui peuvent rester dans le système en temps que professeur. Cette théorie "peut aussi être considérée comme une forme particulièrement accessible des programmes linéaires. Dans le système de Léontief le plus simple, dans lequel aucune substitution d'entrées n'est techniquement possible, la solution optimum est la seule solution efficiente possible. Mais dans des mo- 
dèles plus généraux où les substitions sont possibles, le système peut être déterminé uniquement en résolvant un problème de programmation linéaire formulé de façon convenable ou bien en soumettant la solution à des conditions extérieures restrictives" (18).

La formulation mathématique est simple et correspond à une représentation réaliste du système. La forme des données nécessaires à la construction du modèle est semblable à celle des données que les administrateurs manipulent au cours de leur travail de gestion. Leur compréhension du modèle et leur aptitude à s'en servir s'en trouvent accrues.

Les inconvénients suivants peuvent être cités.

L'une des principales difficultés auxquels se heurtent les modèles d'entrée-sortie tient à la confusion des hypothèses et des objectifs. "Si le modèle ne précise pas les éléments à considérer comme des variables et ceux que l'on doit supposer fixes, il n'apportera qu'une aide infime aux administrateurs du plan effectif. On pourrait citer nombre d'exemples où les rapports numériques élèves-maîtres sont supposés et non incorporés dans le modèle à titre d'objectifs". Cette critique de Dressel P. (19) à l'égard des modèles mathématiques en général se révèle fondée pour les modèles d'entrée-sortie. Oliver R., Hopkins D. et Armacost R. (46) précisent cette critique dans la présentation de leur modèle d'entréesortie appliqué au campus de Berkeley de l'Université de Californie: "Perhaps the single most troublesome aspect of any large scale fixed coefficient model is that there is little opportunity to distinguish institutional variables, administrative controls and environmental parameters from one another, and thus be able to clearly identify the influence of new policies or the relaxation of institutional restrictions upon the structure of the (inputoutput) matrix itself".

D'autre part ce modèle étant général il nécessite une définition et une analyse complète du système. Les données nécessaires à la construction du modèle sont souvent si nombreuses que le temps et l'effort consacrés à la recherche et à la mise en ordre des statistiques risquent de réduire les avantages à attendre de cette méthode.

Cette approche n'est rationnellement utilisable qu'à un niveau agrégé car il faudrait autrement multiplier les matrices d'entrée-sortie et la détermination subséquente des coefficients.

\section{Conclusion}

Pratiquement la plupart des modèles présentés dans cet article se contentent de mesurer le cheminement dans la structure de groupes d'individus relativement homogènes. Les prévisions sont alors de simples extrapolations pondérées par l'évolution actuelle. On peut s'inquiéter du peu d'explication apporté par ces modèles descriptifs au processus de cheminement. Seuls les modèles d'entrée-sortie apportent un certain élément d'explication en introduisant les ressources nécessaires à la réalisation du processus éducatif. Pourtant contrairement au processus classique de production, celui d'acquisition des connaissances dépend également de facteurs individuels (caractéristiques personnelles, comportements psychologiques, relations professeur-étudiants etc...) qui sont ignorés dans un macro-modèle. Indépendamment ou plutôt conjointement aux ressources injectées dans le système, il y aurait lieu d'analyser ces différents facteurs qui conditionnent ou tout au moins influencent fortement le cheminement des étudiants dans la structure. De telles 
approches, outre leur aspect explicatif du processus, permettraient une gestion dynamique du système; les prévisions ainsi élaborées ne seraient plus bâties sur les conséquences mesurables mais sur les causes mêmes du processus de cheminement. En ce sens les simulations de Benson R. et Klasson C. (7) relatives à un système de main d'oeuvre spécialisée sont un premier pas vers cette synthèse des micro et des macro-modèles qui est à notre avis l'orientation actuelle la plus souhaitable pour les modèles de prévision des effectifs étudiants au niveau universitaire.

\section{Bibliographie}

1 Armitage P. et Smith C., "Mise au point et utilisation de modèles exploitables à l'ordinateur pour le système britannique d'enseignement," dans Modèles mathématiques pour la planification de l'enseignement, OCDE, Paris, 1969.

2 Baisuck A., A Projection Model for Higher Educational Systems Planning, Rensselaer Research Corp., Troy, N.Y., May 1968.

3 Baisuck A., "Construction and Evaluation of a Simulation Model for Projecting Higher Education Enrolment," Ph.D. Dissertation, School of Management, Rensselaer Polytechnic Institute, Troy, N.Y., August 1969.

4 Baisuck A. and Wallace W., A Computer Simulation Approach to Enrolment Projection in Higher Education, Rensselaer Research Corp., Troy, N.Y., March 1970.

5 Bartholomew D., Stochastic Models for Social Processes, John Wiley, 1967.

6 Bénard J., "Modèle d'optimisation générale: Economie-Education," dans Modèles mathématiques pour la planification de l'enseignement, OCDE, Paris, 1969, pp. 211-250.

7 Benson, R. and Klasson, C., "A Computer Simulation Model for High-Talent Personnel," in Manpower Planning and Programming, ed., Burack, E. Allyn and Bucon, 1972.

8 Braun H., Hammer G. and Schmid K., "Ein Verfahren zur Ermittlung der Ausbildungskapazität Wissenschaftlicher Hochschulen," Jahrbücher fur Nationalökonomic und Statistik, Vol. 182 (1969), p.p. 381-397.

9 Breneman D., The Stability of Faculty Input Coefficients in Linear Workload Models of the University of California, Ford Foundation Program for Research in University Administration, University of California, April 1969.

10 Caspar R., Bingert A., Bayer W. and Blahusch F., Ein Modell zur Ermittlung der Zulassungszahlen van Studienanfängern an einer Wissenschaftlichen Hochschule, Univer sität Stuttgart, 1968.

11 Caspar R., "Ein Modell Zur Ermittlung Optimaler Zulassungszahlen von Studienanfängern," HISBriefe, Heft, B1, 1969, pp. 2-5.

12 Clough D., A Model for Education-Employment Systems Analysis, University of Toronto, 1967.

13 Clough D. and McReynolds W., "State Transition Model of an Educational System incorporating a Constraint Theory of Supply and Demand," Ontario Journal of Educational Research, Vol. 9, Number 1 (Autumn 1966), pp. 1-18.

14 Corrazini A., The Demand for Higher Education in an Urban Area, Tufts University, Medford, Massachusetts.

15 Correa H., "Modèles mathématiques pour la planification de l'enseignement," dans Modèles mathématiques pour la planification de l'enseignement, OCDE, Paris 1969, pp. 23-95.

16 Dietze H., Studie zur Kapazitätsemittlung an Universitäten, Universität des Saarlandes, 1969.

17 Doermann H., Cross-currents in College Admissions, Teachers College Press, Columbia University, 1968.

18 Dorfman R., Samuelson P. et Solow R., Programmation linéaire et Gestion économique, Dunod, 1962.

19 Dressel P., "Remarques sur l'emploi des modèles mathématiques dans la planification de l'enseignement," dans Modèles mathématiques pour la planification de l'enseignement, OCDE, Paris, 1969. 20 Edwards J. and Orcutt G., "Should Aggregation Prior to Estimation be the Rule?" Review of Economics and Statistics, Vol. LI, No. 4 (November 1969). 
21 Florian M. et Guérin G., “Modèles de prévision de population étudiante,” Rapport technique no. 6, Gresigu, Université de Montréal, mai 1971.

22 Fox T., Long Run Planning for Undergraduate-Higher Education Capacity Needs: Basing Enrolment Projections on Partial-College-Potential vs Full-College-Potential, Institute for Research on Human Resources, Pennsylvania State University, University Park, Penn., August 1970.

23 Gani J., "Formulae for Projecting Enrolments and Degrees Awarded in Universities," Journal of Royal Statistics Society, Series A, Vol. 126, Part 3 (1963), pp. 400-409.

24 Gordon P., Théorie des chaînes de Markov finies et ses applications, Dunod, 1965.

25 Guérin, G., "Prévision des effectifs étudiants dans un secteur d'étude d'une institution universitaire," Gresigu, Université de Montréal, numéro 19 (mai 1972).

26 Guérin G., "Elaboration d'un modèle de prévision des effectifs étudiants au niveau universitaire," thèse de doctorat non publié, département d'Informatique, Université de Montréal, septembre 1972.

27 Guérin G., "Structuration du système universitaire," Stoa, HII-1 (1973), pp. 29-46.

28 Hall A., "Projecting University Populations," Vestes (Aust. Univ. Rev.), Vol. 5, no. 3 (1962), pp. 66-73.

29 Hoenack S., "Efficient Allocation of Subsidies for College Student Institute for Defense Analysis," Presented at Symposium on Operations Analysis of Education, Washington, D.C., November 1967. 30 Jacoby E., Methods of School Enrolment Projection, U.N.E.S.C.O., Paris, France, August 1959.

31 Jewett J., College Admissions Planning: Use of a Student Segmentation Model, Paper P-23, Ford Foundation for Research in University Administration, University of California, November 1971.

32 Karlin S., Initiation aux processus aléatoire, Dunod, 1969.

33 Koenig H., Systems Models and their Applications in Management Planning and Resource Allocation in Institutions of Higher Education, Division of Engineering Research, Michigan State University, November 1969.

34 Lawson D. and Jewett F., An Input-Output Model of Humboldt State College, Humboldt State College, 1969.

35 Lins L., Methodology of Enrolment Projections for Colleges and Universities, Association of American College Registrars and Admissions Officers, 1960.

36 Marshall K. and Oliver R., A Constant Work Model for Student Attendance and Enrolment, Report No. 69-1, Ford Foundation Program for Research in University Administration, University of California, February 1969.

37 Marshall K., Oliver R. and Suslow S., Under-Graduate Enrolments and Attendance Patterns, Administration Studies Project in Higher Education, University of California, March 1970.

38 Menges G. and Elstermann G., Forecasting and Planning the Structure of Personnel of a University, NATO Conference on Manpower Planning, Porto, Portugal, September 1969.

39 Menges G. and Elstermann G., Capacity Models in University Management, XVII International Conference of TIMS, London, April 1970.

40 Moser C. and Redfern P., "Education and Manpower: Some Current Research," in C. Berners-Lee, (ed.), Models for Decision, English Universities Press, London, 1965.

41 Muetira M., The Social Demand for Higher Education in Portugal, N.A.T.O. Conference on Manpower Planning, Porto, Portugal, September 1969.

42 Nordell L., Dynamic Input-Output Model of the California Educational System, Technical Report No. 25, Center for Research in Management Science, University of California, Berkeley, August 1967.

43 Office of Program Planning and Evaluation, Students and Building, An analysis of Selected Federal Programs for Higher Education, USOE, Washing ton, D.C., OE-0054, May 1968.

44 O'Grady W. and Feddersen A., A Student Flow Model for California State College, Fullerton: A Feasibility Study, Division of Analytic Studies, the California State Colleges, Los Angeles, January 1972.

45 Oliver R., Models for Predicting Gross Enrolments at the University of California, Research Report No. 68-3, Ford Foundation Program for Research in University Administration, University of California, August 1968.

46 Oliver R., Hopkins D. and Armacost R., An Academic Productivity and Planning.Model for a Uni- 
versity Campus, Administrative Studies Project in Higher Education, University of California, Berkeley, California, February 1970.

47 Price W., Les modèles mathématiques de systèmes de gestion de personnel, Département de Mechanical Engineering, Royal Military College, Kingston, Ontario, October 1969.

48 Radner R. and Miller L., Resource Requirements for a Universal Two-year College Program, Working Paper Number 2, Carnegie Commission on the Future of Higher Education, Berkeley, California, 1969.

49 Redfern P., Input-Output Analysis and its Application to Education and Manpower Planning, Her Majesty's Stationery Office, London 1967.

50 Smith W., Review of a Student Flow Model, University of California, Los Angeles, April 1969.

51 Smith W., A Student Flow Model, Office of Advanced Planning at the University of California at Los Angeles, Los Angeles, California 1970.

52 Stone R., "A Model for the Educational System," Minerva, Vol. 3 (1965), pp. 172-186.

53 Stone R., "Aperçu général de la Conférence," dans Modèles mathématiques pour la planification de l'enseignement, OCDE, Paris 1968, pp. 7-22.

54 Thonstad T., Education and Manpower, University of Toronto Press, 1968.

55 Thonstad T., "A Mathematical Model of Norwegian Educational System," in Mathematical Models in Educational Planning, OCDE, Paris, 1967, pp. 125-158.

56 Turksen I., A Micro Level Forecasting Model of Student Enrolment, Department of Industrial Engineering, University of Toronto, April 1970.

57 Weathersby G. and Weinstein M., A Structural Comparison of Analytical Models for University Planning, Paper P-12, Ford Foundation Program for Research in University Administration, University of California, August 1970.

58 Weitz H., An Assessment of the Contribution of Operations Research to Educational Planning, IBM Yorktown Heights, N.Y., June 1969.

59 Weitz H., Bibliography for Operations Analysis of Education, IBM Yorktown Heights, N.Y., 1969.

60 White L., Mathematical Programming Models for Determining Freshman Scholarship Offers, Sloan Working Paper No. 379-69, Massachusetts Institute of Technology, March 1969.

61 WICHE, Program Classification Structure, Technical Report No. 13, 1970.

62 Wolfman B. and Fong P., Construction of Student Fees at the University of California, Office of the President, Berkeley, May 1968.

63 Wurtele Z., Mathematical Models for Educational Planning, System Development Corporation, Santa Monica, California, November 1967.

64 Young A. and Almond G., "Predicting Distributions of Staff," Computer Journal, 3, No. 4 (1961), pp. 246-250. 